\title{
A BRIGHT SUBMILLIMETER SOURCE IN THE BULLET CLUSTER (1E0657-56) FIELD DETECTED WITH
} BLAST

\author{
Marie Rex ${ }^{1}$, Peter A. R. Ade ${ }^{2}$, Itziar AretXaga ${ }^{3}$, James J. Bock ${ }^{4,5}$, Edward L. Chapin ${ }^{6}$, Mark J. Devlin ${ }^{1}$, \\ Simon R. Dicker ${ }^{1}$, Matthew Griffin ${ }^{2}$, Joshua O. Gundersen ${ }^{7}$, Mark Halpern $^{6}$, Peter C. Hargrave ${ }^{2}$, David H. Hughes ${ }^{3}$, \\ Jeff Klein $^{1}$, Gaelen Marsden ${ }^{6}$, Peter G. Martin ${ }^{8,9}$, Philip Mauskopf $^{2}$, Alfredo Montaña ${ }^{3}$, Calvin B. Netterfield ${ }^{9,10}$, \\ Luca Olmi $^{11,12}$, Enzo Pascale ${ }^{2}$, Guillaume Patanchon ${ }^{13}$, Douglas Scott ${ }^{6}$, Christopher Semisch $^{1}$, Nicholas Thomas $^{7}$, \\ Matthew D. P. Truch ${ }^{1}$, Carole Tucker ${ }^{2}$, Gregory S. Tucker ${ }^{14}$, Marco P. Viero ${ }^{9}$, and Donald V. Wiebe ${ }^{6,10}$ \\ ${ }^{1}$ Department of Physics \& Astronomy, University of Pennsylvania, 209 South 33rd Street, Philadelphia, PA 19104, USA; madamson@physics.upenn.edu \\ ${ }^{2}$ Department of Physics \& Astronomy, Cardiff University, 5 The Parade, Cardiff, CF24 3AA, UK \\ ${ }^{3}$ Instituto Nacional de Astrofísica Óptica y Electrónica (INAOE), Aptdo. Postal 51 y 72000 Puebla, Mexico \\ ${ }^{4}$ Jet Propulsion Laboratory, Pasadena, CA 91109-8099, USA \\ ${ }^{5}$ Observational Cosmology, MS 59-33, California Institute of Technology, Pasadena, CA 91125, USA \\ ${ }^{6}$ Department of Physics \& Astronomy, University of British Columbia, 6224 Agricultural Road, Vancouver, BC V6T 1Z1, Canada \\ ${ }^{7}$ Department of Physics, University of Miami, 1320 Campo Sano Drive, Carol Gables, FL 33146, USA \\ ${ }^{8}$ Canadian Institute for Theoretical Astrophysics, University of Toronto, 60 St. George Street, Toronto, ON M5S 3H8, Canada \\ ${ }^{9}$ Department of Astronomy \& Astrophysics, University of Toronto, 50 St. George Street, Toronto, ON M5S 3H4, Canada \\ ${ }^{10}$ Department of Physics, University of Toronto, 60 St. George Street, Toronto, ON M5S 1A7, Canada \\ ${ }^{11}$ Istituto di Radioastronomia, Largo E. Fermi 5, I-50125, Firenze, Italy \\ 12 University of Puerto Rico, Rio Piedras Campus, Physics Department, Box 23343, UPR Station, San Juan, Puerto Rico \\ ${ }^{13}$ Laboratoire APC, 10, rue Alice Domon et Léonie Duquet, 75205 Paris, France \\ 14 Department of Physics, Brown University, 182 Hope Street, Providence, RI 02912, USA \\ Received 2009 April 7; accepted 2009 July 29; published 2009 August 27
}

\begin{abstract}
We present the 250,350, and $500 \mu \mathrm{m}$ detection of bright submillimeter emission in the direction of the Bullet Cluster measured by the Balloon-borne Large-Aperture Submillimeter Telescope (BLAST). The $500 \mu \mathrm{m}$ centroid is coincident with an AzTEC $1.1 \mathrm{~mm}$ point-source detection at a position close to the peak lensing magnification produced by the cluster. However, the $250 \mu \mathrm{m}$ and $350 \mu \mathrm{m}$ centroids are elongated and shifted toward the south with a differential shift between bands that cannot be explained by pointing uncertainties. We therefore conclude that the BLAST detection is likely contaminated by emission from foreground galaxies associated with the Bullet Cluster. The submillimeter redshift estimate based on 250-1100 $\mu \mathrm{m}$ photometry at the position of the AzTEC source is $z_{\text {phot }}=2.9_{-0.3}^{+0.6}$, consistent with the infrared color redshift estimation of the most likely Infrared Array Camera counterpart. These flux densities indicate an apparent far-infrared (FIR) luminosity of $L_{\mathrm{FIR}}=2 \times 10^{13} L_{\odot}$. When the amplification due to the gravitational lensing of the cluster is removed, the intrinsic FIR luminosity of the source is found to be $L_{\mathrm{FIR}} \leqslant 10^{12} L_{\odot}$, consistent with typical luminous infrared galaxies.
\end{abstract}

Key words: galaxies: clusters: individual (1E0657-56) - submillimeter

Online-only material: color figure

\section{INTRODUCTION}

A decade of advances in submillimeter surveys has led to a broad understanding of the galaxies responsible for the highredshift component of the cosmic infrared background (Smail et al. 1997; Barger et al. 1998; Hughes et al. 1998; Webb et al. 2003; Greve et al. 2004; Pope et al. 2006; Coppin et al. 2006; Bertoldi et al. 2007; Knudsen et al. 2008; Devlin et al. 2009). These massive submillimeter galaxies (SMGs) are considered to be the products of galaxy mergers and are associated with a vital stage of galaxy evolution. With typical far-infrared (FIR) luminosities $>10^{12} L_{\odot}$, they are presumed to be the high-redshift counterparts to (ultra)luminous infrared galaxies (LIRGs, ULIRGs). The source of this energy is generally attributed to powerful starbursts, suggesting star formation rates of 100-1000 $M_{\odot} \mathrm{yr}^{-1}$. Approximately half of these galaxies are located at $1.9 \lesssim z \lesssim 2.9$ (Chapman et al. 2005; Aretxaga et al. 2007), constituting a significant component of the star formation rate at this epoch (Pérez-González et al. 2005).

The increased mapping speeds of current submillimeter instruments have facilitated recent large-area blank-field surveys (Siringo et al. 2009; Devlin et al. 2009; Austermann et al. 2009).
These surveys provide a large statistical sample of bright SMGs. However, resolution restricts the depth to the confusion limit and they are unable to probe the abundant faint end of the number counts. Observations directed toward massive galaxy clusters take advantage of gravitational lensing to penetrate the confusion limit and sample intrinsically fainter and higher-redshift SMGs (Smail et al. 1997; Zemcov et al. 2007; Knudsen et al. 2008).

An extremely bright millimeter source was recently reported from a survey of the Bullet Cluster conducted with the $1.1 \mathrm{~mm}$ AzTEC receiver on the Atacama Submillimeter Telescope Experiment (ASTE; Wilson et al. 2008). Its millimeter properties are given in Table 1. The high apparent flux and close proximity to a critical line of magnification on the lensing model motivated the authors to conclude that the source is likely to be a background galaxy amplified by the foreground mass. This AzTEC source (MMJ065837-5557.0) is coincident with a LIRG detected by the Infrared Array Camera (IRAC; which possibly has multiple infrared images of the galaxy), with a redshift $z_{\text {phot }}^{\mathrm{IR}}=2.7 \pm 0.2$ (Wilson et al. 2008; Gonzalez et al. 2009).

We present the 250, 350, and $500 \mu \mathrm{m}$ measurements of a bright source (BLAST J065837-555708) located in the 
Table 1

Millimeter Properties of AzTEC Source MMJ065837-5557.0

\begin{tabular}{lc}
\hline \hline \multicolumn{1}{c}{ Property } & Value \\
\hline Flux density & $13.5 \pm 1.0 \mathrm{mJy}$ \\
R.A. centroid position & $6^{\mathrm{h}} 58^{\mathrm{m}} 37^{\mathrm{s}} .31(\mathrm{~J} 2000)$ \\
Decl. centroid position & $-55^{\circ} 57^{\prime} 1^{\prime \prime} .5(\mathrm{~J} 2000)$
\end{tabular}

Note. $1.1 \mathrm{~mm}$ flux density and centroid position of MMJ065837-5557.0 from Wilson et al. (2008).

direction of the Bullet Cluster. With significances $6 \sigma, 5 \sigma$, and $9 \sigma$ at 250,350 , and $500 \mu \mathrm{m}$, respectively, this detection is the highest signal-to-noise source in the $0.25 \mathrm{deg}^{2}$ map of the region taken with the Balloon-borne Large-Aperture Submillimeter Telescope (BLAST) in 2006 (Pascale et al. 2008). Details of the observations and data reduction techniques are presented in Section 2. Extracted photometry as well as a photometric redshift estimate and constraints on the intrinsic properties of the millimeter source are given in Section 3. Finally, our conclusions are summarized in Section 4.

\section{OBSERVATIONS AND REDUCTION}

BLAST conducted an 11 day high-altitude flight from McMurdo Station, Antarctica, in December of 2006 (BLAST06). The telescope has a $1.8 \mathrm{~m}$ primary mirror and three large-format bolometer arrays that image the sky simultaneously at 250,350 , and $500 \mu \mathrm{m}$ over a $14^{\prime} \times 7^{\prime}$ field of view. The telescope pointspread function (PSF) at each wavelength is best fitted by Gaussians with full widths at half-maximum (FWHMs) $36^{\prime \prime}, 42^{\prime \prime}$, and $60^{\prime \prime}$ at 250,350 , and $500 \mu \mathrm{m}$, respectively. BLAST scans the sky quickly $\left(\sim 0.1 \mathrm{deg} \mathrm{s}^{-1}\right)$ in azimuth, while drifting slowly in elevation. Details of the BLAST instrument can be found in Pascale et al. (2008). The BLAST wavelengths were selected to sample the peak of the spectral energy distribution (SED) for high-redshift starburst galaxies as well as cooler star-forming regions within our own Galaxy.

A large portion of the BLAST06 flight ( $>150 / 220 \mathrm{hr}$ ) was dedicated to conducting large-area, wide and deep blank-field surveys to identify and characterize the high-redshift population of starburst galaxies that comprise the FIR extragalactic background (Devlin et al. 2009). Two smaller fields were also targeted which are known to contain massive galaxy clusters, including a $1.1 \mathrm{deg}^{2}$ map around Abell 3112 and a $0.25 \mathrm{deg}^{2}$ map around the Bullet Cluster. The Bullet Cluster field was visited three times during the flight, with a total observing time of $2.0 \mathrm{hr}$.

The raw BLAST06 time-stream data are reduced using a common pipeline detailed in Pascale et al. (2008) and Truch et al. (2008). The absolute calibration of the BLAST06 data is determined based on observations of the star VY CMa with $10 \%-13 \%$ uncertainty which is highly correlated between bands (Truch et al. 2009). Signal and variance maps of the Bullet Cluster with $10^{\prime \prime}$ pixels are generated using the mapmaking algorithm OptBin (E. Pascale et al. 2009, in preparation). The rotation of the sky between visits to the field provided $\sim 10^{\circ}$ cross-linking between azimuthal scans. This shallow angle is insufficient to constrain low frequency drifts in the detectors and preliminary maps suffered from significant striping along the telescope scan direction. In order to suppress these artifacts, the time-stream data are filtered by the map maker, whitening the map on scales $>16^{\prime}$. The appropriate PSF for each band is produced by stacking maps of VY Cma, co-added with the proper sky rotation, and filtered in the same man-
Table 2

Peak Positions of BLAST J065837-555708

\begin{tabular}{|c|c|c|c|c|}
\hline \multirow[b]{2}{*}{$\begin{array}{c}\lambda \\
(\mu \mathrm{m}) \\
\end{array}$} & \multicolumn{2}{|c|}{ Before Correction } & \multicolumn{2}{|c|}{ After Correction } \\
\hline & $\begin{array}{c}\text { Centroid R.A. } \\
\text { (h:m:s) }\end{array}$ & $\begin{array}{l}\text { Centroid Decl. } \\
\text { (d:m:s) }\end{array}$ & $\begin{array}{c}\text { Centroid R.A. } \\
\text { (h:m:s) }\end{array}$ & $\begin{array}{c}\text { Centroid Decl. } \\
\text { (d:m:s) }\end{array}$ \\
\hline 250 & $06: 58: 35.3$ & $-55: 57: 08$ & $06: 58: 36.7$ & $-55: 57: 33$ \\
\hline 350 & $06: 58: 36.2$ & $-55: 56: 58$ & $06: 58: 36.6$ & $-55: 57: 22$ \\
\hline 500 & $06: 58: 36.0$ & $-55: 56: 44$ & $06: 58: 37.3$ & $-55: 57: 09$ \\
\hline
\end{tabular}

Notes. Positions of the peaks in each band associated with BLAST J065837-555708. The positions are given before and after the correctional offset applied based on stacking the $250 \mu \mathrm{m}$ map against $24 \mu \mathrm{m}$ sources.

ner as the final maps to enable accurate source extraction and calibration. ${ }^{15}$ A catalog of sources for each band is compiled using a source-finding algorithm which selects the peaks in a smoothed map produced by the noise-weighted convolution of the image with the PSF. The peak positions in each band associated with BLAST J065837-555708 are given in Table 2.

The telescope pointing solution is reconstructed postflight by combining $100 \mathrm{~Hz}$ rate gyroscope data with $\sim 1 \mathrm{~Hz}$ star camera solutions which provide an absolute reference (Pascale et al. 2008). The star cameras are mounted to the same rigid structure as the telescope. Nevertheless, tilting the structure in elevation results in small changes to the angular offset between the boresights. To correct this effect, bright compact objects were targeted as pointing calibrators at different elevations throughout the flight. The resulting $1 \sigma$ pointing uncertainty over the elevation range of observed calibrators $\left(30^{\circ}-50^{\circ}\right)$ is $\sim 5^{\prime \prime}$. Observations of the Bullet Cluster were performed at high elevation $\left(\sim 54^{\circ}\right)$, outside the range of our pointing calibrators. They were also conducted early in the flight, before the telescope focus had been finalized. While these factors lead us to suspect larger absolute pointing uncertainties for sources in the Bullet Cluster field, the optical design of BLAST does not allow for relative offsets between the bands.

The absolute pointing offset for the BLAST maps of the Bullet Cluster is determined by stacking them against positions of $24 \mu \mathrm{m}$ sources. This $24 \mu \mathrm{m}$ catalog contains sources with a significance $\geqslant 3 \sigma$ extracted from the Spitzer MIPS observations of the field using the APEX source-finding algorithm (Makovoz et al. 2002). At $250 \mu \mathrm{m}$, this analysis produces the highest signal to noise, indicating a $27^{\prime \prime}$ astrometry correction required for the BLAST maps. The corrected peak positions of BLAST J065837-555708 are given in Table 2.

\section{ANALYSIS}

The Bullet Cluster, at redshift $z=0.297$ (Tucker et al. 1998), is the product of two merging galaxy clusters. The less massive member has passed through the dominant mass with a relative velocity of $\gtrsim 4500 \mathrm{~km} \mathrm{~s}^{-1}$. This extreme interaction emphasizes the displacement between the mass of the cluster, traced by weak lensing models, and the hot X-ray gas, providing dramatic evidence for the presence of dark matter (Clowe et al. 2004). Figure 1 shows maps of the Bullet Cluster at optical, X-ray, and infrared wavelengths. The image in the left-hand panel combines optical maps from Magellan and the Hubble Space Telescope (HST; Clowe et al. 2004) with Chandra X-ray data (Markevitch et al. 2002). The X-ray emission reveals hot gas that represents the majority of the baryonic matter in the cluster

\footnotetext{
15 Maps and PSFs for the field are available to download from http://blastexperiment.info.
} 


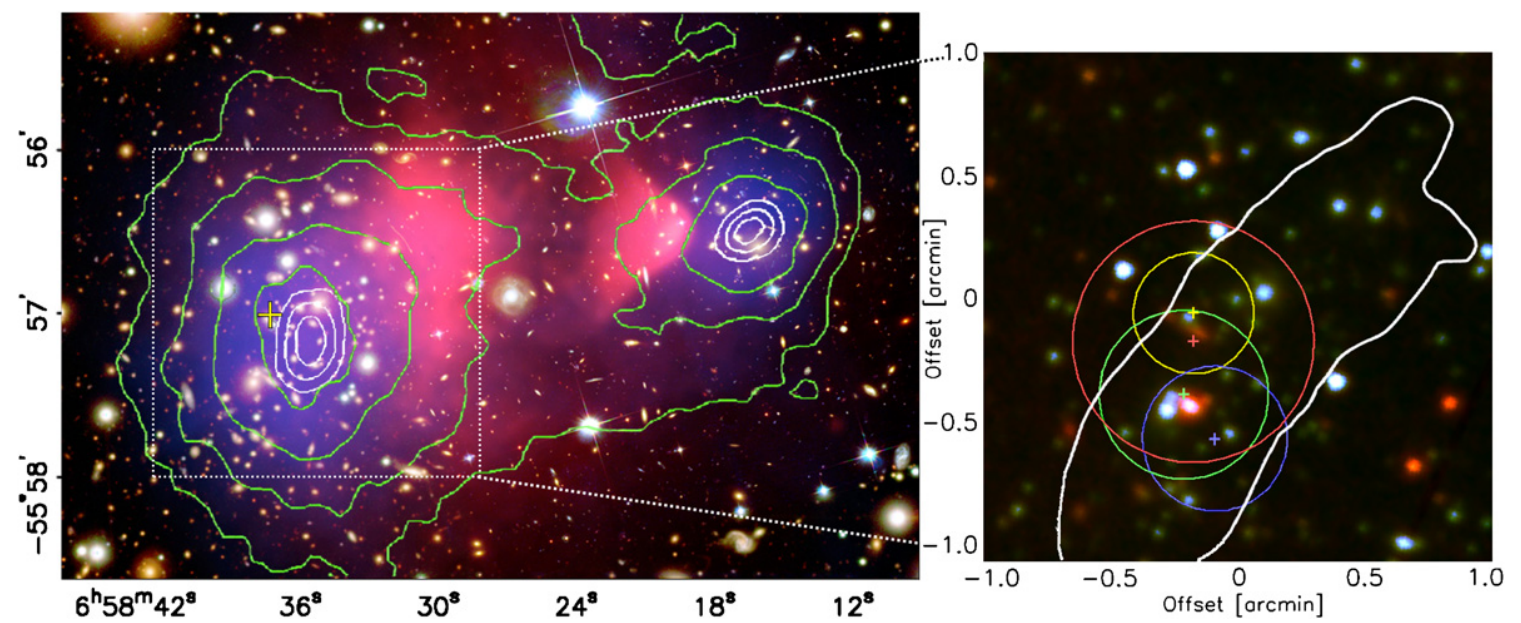

Figure 1. Left panel: background image combines optical maps from Magellan and HST (Clowe et al. 2004) with Chandra X-ray data (Markevitch et al. 2002). The $\mathrm{X}$-ray map reveals the hot gas which represents the majority of the baryonic matter in the cluster (shown in pink). The distribution of the dark matter is shown in blue, with weak lensing contours overplotted in green. Image based on Figure 1 in Clowe et al. (2006). The yellow cross indicates the position of the AzTEC detection. Right panel: a $2^{\prime} \times 2^{\prime}$ detail based on IRAC 3.6, 4.5, and $8.0 \mu \mathrm{m}$ maps of the region. The BLAST 250,350 , and $500 \mu \mathrm{m}$ positions are shown in blue, green, and red, respectively. The AzTEC $1.1 \mathrm{~mm}$ position is shown in yellow. The crosses indicate the centroids of the detections and the circles represent the FWHM of each beam. The white contour denotes the critical curve corresponding to infinite magnification based on HST data (Bradač et al. 2006).

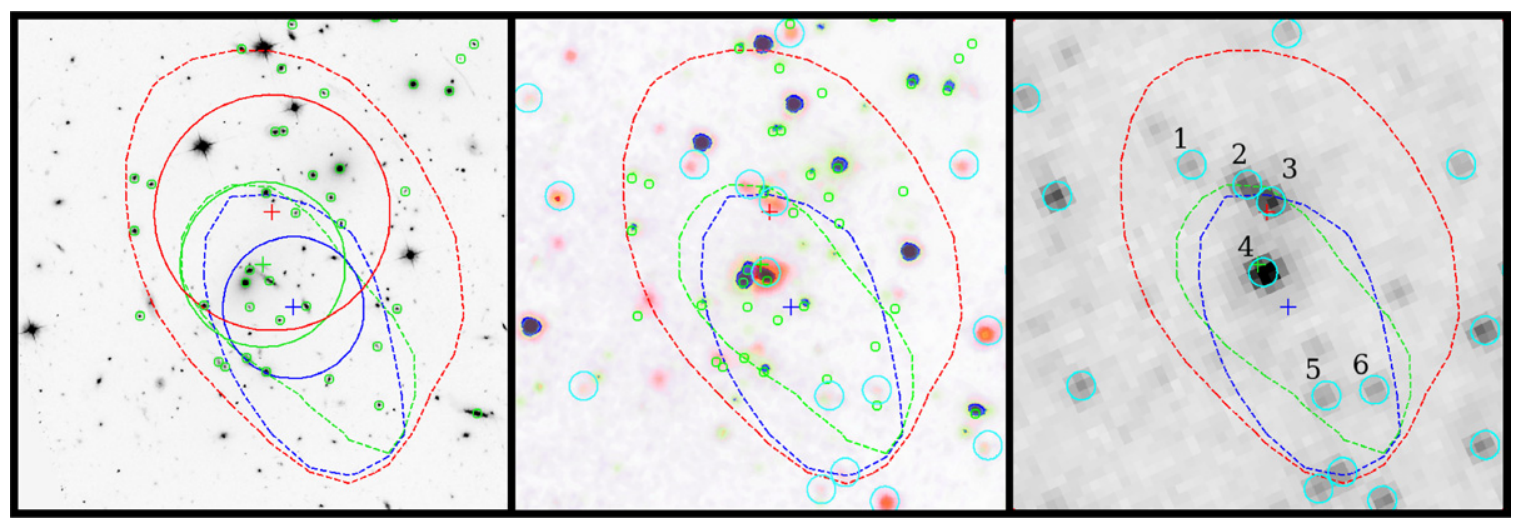

Figure 2. Detailed $2^{\prime} \times 2^{\prime}$ region around BLAST J065837-555708. The BLAST 250, 350, and $500 \mu \mathrm{m}$ positions are shown in blue, green, and red, respectively. Crosses indicate the centroids of the measurements and corresponding solid circles represent the FWHM of the beam. Dashed lines show $3 \sigma$ contours for each BLAST detection. Left-hand panel: HST $606 \mathrm{~nm}$ map. Small green circles indicate the positions of spectroscopically verified cluster members (Mehlert et al. 2001). Many of these foreground galaxies may be contributing flux to the submillimeter measurement and shifting the centroid positions away from the position of the background LIRG. Middle panel: red, green, and blue composite image made from IRAC 8.0, 4.5, and $3.6 \mu \mathrm{m}$ maps, respectively. Again, cluster members are indicated with small green circles. $24 \mu \mathrm{m}$ counterparts detected with a significance $>5 \sigma$ are marked in cyan. The infrared maps reveal a number of bright galaxies near the peak of the $350 \mu \mathrm{m}$ emission. Right-hand panel: MIPS $24 \mu \mathrm{m}$ map. Cyan circles indicate $>5 \sigma$ sources. Sources within the $3 \sigma$ BLAST contours are numbered and the extracted infrared photometry is given in Table 3. Sources 2 and 3 are likely split images of the lensed background LIRG associated with the $1.1 \mathrm{~mm}$ detection. They coincide with the peak of the $500 \mu \mathrm{m}$ emission, although the shorter wavelength peaks fall closer to source 4 . This lower-redshift source is likely to be brighter at short BLAST wavelengths, contributing significantly to the emission in those bands. Sources 1,5 , and 6 , which also appear at $24 \mu \mathrm{m}$ may additionally contribute at BLAST wavelengths. The elongation of the contours could be explained by confusion of these sources in the lower resolution BLAST maps.

(shown in pink). The distribution of the dark matter is shown in blue with weak lensing contours overplotted in green (Clowe et al. 2006). The yellow cross marks the position of the AzTEC point-source detection at $1.1 \mathrm{~mm}$, near the peak of the largest mass distribution.

The right-hand panel of Figure 1 shows a $2^{\prime} \times 2^{\prime}$ detail of the region. The color image is produced from Spitzer IRAC 3.6, 4.5, and $8.0 \mu \mathrm{m}$ maps. The BLAST centroids at 250, 350, and $500 \mu \mathrm{m}$ are shown in blue, green, and red, respectively. The AzTEC $1.1 \mathrm{~mm}$ position is shown in yellow. Each cross indicates the position of the peak BLAST detection, with circles representing the FWHM of each beam. It is apparent that the relative offsets between the BLAST beams is inconsistent with the $5^{\prime \prime}$ pointing error determined in other BLAST06 maps (Pascale et al. 2008; Devlin et al. 2009). We therefore consider the possibility that the centroids are shifted due to emission from a combination of galaxies at different redshifts, slightly displaced along the line of sight.

\subsection{Photometry}

The displacement of the BLAST centroid positions from the location of the millimeter source increases with frequency and detections are elongated in the direction of that displacement. These features are illustrated in Figure 2, showing a detailed $2^{\prime} \times$ $2^{\prime}$ region around BLAST J065837-555708. The differential shift between bands suggests emission from other galaxies with small angular offsets to the line of sight, south of the AzTEC source. The central panel of Figure 2 is a composite of IRAC 3.6, 4.5, and $8.0 \mu \mathrm{m}$ images. The maps reveal multiple cluster 
Table 3

Infrared Photometry for $24 \mu \mathrm{m}$ Sources Associated with BLAST J065837-555708

\begin{tabular}{|c|c|c|c|c|c|c|c|}
\hline ID & $\begin{array}{c}24 \mu \mathrm{m} \text { R.A. } \\
(\mathrm{hr})\end{array}$ & $\begin{array}{c}24 \mu \mathrm{m} \text { Decl. } \\
(\mathrm{deg})\end{array}$ & $\begin{array}{r}S_{3.6} \\
(\mu \mathrm{Jy})\end{array}$ & $\begin{array}{c}S_{4.5} \\
(\mu \mathrm{Jy})\end{array}$ & $\begin{array}{c}S_{5.8} \\
(\mu \mathrm{Jy})\end{array}$ & $\begin{array}{c}S_{8.0} \\
(\mu \mathrm{Jy})\end{array}$ & $\begin{array}{c}S_{24} \\
(\mu \mathrm{Jy})\end{array}$ \\
\hline 1 & $6: 58: 39.69$ & $-55: 56: 56$ & $18.6 \pm 0.2$ & $26.2 \pm 0.3$ & $70.6 \pm 1.1$ & $40.0 \pm 1.9$ & $252.7 \pm 37.2$ \\
\hline 2 & 6:58:37.99 & $-55: 57: 01$ & $19.1 \pm 0.2$ & $25.7 \pm 0.3$ & $76.5 \pm 1.1$ & $60.5 \pm 1.9$ & $596.2 \pm 34.9$ \\
\hline 3 & $6: 58: 37.21$ & $-55: 57: 06$ & $28.5 \pm 0.2$ & $49.0 \pm 0.3$ & $69.2 \pm 1.1$ & $86.9 \pm 2.0$ & $840.4 \pm 36.4$ \\
\hline 4 & $6: 58: 37.40$ & $-55: 57: 24$ & $309.6 \pm 0.5$ & $269.5 \pm 0.5$ & $190.4 \pm 1.3$ & $528.3 \pm 2.1$ & $1482.0 \pm 34.8$ \\
\hline 5 & $6: 58: 35.62$ & $-55: 57: 55$ & $72.0 \pm 0.2$ & $47.4 \pm 0.3$ & $60.2 \pm 1.1$ & $25.6 \pm 1.9$ & $306.4 \pm 37.4$ \\
\hline 6 & 6:58:34.09 & $-55: 57: 54$ & $32.5 \pm 0.2$ & $23.0 \pm 0.3$ & $41.9 \pm 1.1$ & $18.4 \pm 1.9$ & $285.0 \pm 36.5$ \\
\hline
\end{tabular}

Note. IR photometry corresponding to the $24 \mu \mathrm{m}$ sources located within the BLAST $3 \sigma$ contours which have a significance of $>5 \sigma$.

Table 4

Submillimeter Flux Densities of MMJ065837-5557.0

\begin{tabular}{|c|c|c|c|c|}
\hline $\begin{array}{c}\lambda \\
(\mu \mathrm{m}) \\
\end{array}$ & $\begin{array}{l}\text { Measured Flux } \\
(\mathrm{mJy})\end{array}$ & $\begin{array}{l}\text { SZE } \\
(\mathrm{mJy})\end{array}$ & $\begin{array}{l}\text { SZE-Corrected Flux } \\
(\mathrm{mJy})\end{array}$ & $\begin{array}{l}\text { Color-Corrected Flux } \\
(\mathrm{mJy})\end{array}$ \\
\hline 50 & $91 \pm 27$ & $0.15 \pm 0.03$ & $91 \pm 27$ & $94 \pm 30$ \\
\hline 350 & 95 & 1.1 & & 96 \\
\hline 500 & $121 \pm 16$ & $10.6 \pm 1.7$ & $110 \pm 16$ & $110 \pm 21$ \\
\hline
\end{tabular}

Notes. Flux densities in each band associated with BLAST J065837-555708 at the position of the AzTEC detection. The contribution from SZE is calculated at the position of the AzTEC point source, based on the theoretical model outlined in Wilson et al. (2008). This small contamination is subtracted from the measured fluxes for further analysis. The monochromatic flux densities based on the best-fit SED are given in the final column.

member galaxies that may be contributing flux to the BLAST submillimeter measurements and shifting the centroid positions away from the background LIRG. For a galaxy contained within the Bullet Cluster $(z \sim 0.3)$, BLAST colors sample the Rayleigh-Jeans side of the SED. This emission scales as $S_{v} \sim v^{2+\beta}$, and would contribute to the BLAST bands in a form consistent with the observations. The MIPS $24 \mu \mathrm{m}$ map is shown in the right-hand panel of Figure 2, with cyan circles around $>5 \sigma$ sources. Those located within the $3 \sigma$ flux density contours of the BLAST emission are numbered and infrared photometry extracted for each using the APEX pipeline is given in Table 3. Though a number of these galaxies likely contribute to the submillimeter detection, sources 2,3 , and 4 clearly dominate the emission at $24 \mu \mathrm{m}$ and line up with the centroid peaks of the submillimeter maps. Sources 2 and 3 are likely double images of the lensed background LIRG (Wilson et al. 2008; Gonzalez et al. 2009), while source 4 is a spectroscopically confirmed cluster member (Mehlert et al. 2001). We estimate the submillimeter flux densities of each of the lensed high-redshift millimeter source detected by AzTEC and the foreground dominant cluster member galaxy by simultaneously fitting the PSF to the BLAST maps at the positions of both sources using the maximumlikelihood algorithm presented in Scott et al. (2002). Because the mid-infrared images of the lensed LIRG are closely spaced compared to the scale of the resolution, we adopt the millimeter position of Wilson et al (2008) for this component in the jointfit. The submillimeter photometry of the LIRG and the bright cluster member galaxy are given in Tables 4 and 5, respectively. The measured $500 \mu \mathrm{m}$ emission is dominated by an association with the millimeter AzTEC source. However, our estimates of the shorter wavelength contributions to the flux densities at 250 and $350 \mu \mathrm{m}$ favor the cluster member galaxy as the dominant source of emission. Consequently, the observed submillimeter spectrum of the background LIRG is flattened across the BLAST bands.
Table 5

Submillimeter Flux Densities of the Dominant Cluster Member Galaxy

\begin{tabular}{|c|c|c|c|c|}
\hline $\begin{array}{c}\lambda \\
(\mu \mathrm{m}) \\
\end{array}$ & $\begin{array}{c}\text { Measured Flux } \\
(\mathrm{mJy})\end{array}$ & $\begin{array}{l}\text { SZE } \\
(\mathrm{mJy})\end{array}$ & $\begin{array}{c}\text { SZE-Corrected Flux } \\
(\mathrm{mJy})\end{array}$ & $\begin{array}{l}\text { Color-Corrected Flux } \\
(\mathrm{mJy})\end{array}$ \\
\hline 50 & $129 \pm 26$ & $0.13 \pm 0.03$ & $129 \pm 26$ & $131 \pm 30$ \\
\hline 350 & $104 \pm 23$ & $1.0 \pm 0.2$ & $103=$ & 106 \\
\hline 500 & $49 \pm 18$ & $9.4 \pm 1.5$ & $40 \pm 18$ & $38 \pm 18$ \\
\hline
\end{tabular}

Notes. Submillimeter flux densities in each band associated with the dominant $24 \mu \mathrm{m}$ cluster member galaxy at R.A. $(\mathrm{J} 2000)=6: 58: 37.40$, decl. $(\mathrm{J} 2000)=$ $-55: 57: 24$, identified as source 4 in Figure 2 and Table 3. The columns are analogous to those in Table 4.

Submillimeter emission in this region is not confined to compact sources. IRAS maps reveal foreground cirrus emission of $5 \mathrm{MJy} \mathrm{sr}^{-1}$ at $100 \mu \mathrm{m}$. However, this structure is smooth on scales of $\lesssim 1^{\circ}$ and therefore filtered out of the BLAST maps. The measured BLAST flux densities are adjusted to account for a small contamination by the thermal Sunyaev-Zel'dovich effect (SZE). This excess flux density results from the hot gas in the cluster boosting cosmic microwave background photons to higher energies through inverse Compton scattering. Following Wilson et al. (2008), we estimate the SZE flux contribution to the BLAST bands at the position of the AzTEC detection by developing a model to describe the electron density distribution of the Bullet Cluster. We assume a two-component spherically symmetric $\beta_{\text {gas }}$-model $\left(\beta_{\text {gas }}=0.7\right)$ and a range of total cluster mass, $M_{\mathrm{tot}}=(1.5-2.1) \times 10^{15} M_{\odot}$, distributed in a 3:1 ratio (Nusser 2008) between the main and subclusters. Each component is centered at the peak of the corresponding observed X-ray surface brightness distribution given by Clowe et al. (2006). Provided that the electron temperature is similar to the X-ray gas temperature, and assuming each component is isothermal, we estimate electron temperatures $\sim 15.4-16.3 \mathrm{keV}$ for the main cluster and $\sim 6.0-7.4 \mathrm{keV}$ for the subcluster, in agreement with the temperature map provided by Markevitch et al. (2002). Relativistic corrections were applied using the analytical approximation given by Itoh et al. (1998). The results of these simulations and our revised source flux densities are given in Table 4. We have also used this model to estimate the SZE at the position of the bright cluster member galaxy and these results are shown in Table 5.

\subsubsection{Photometric Redshifts}

The shape of the SED in the submillimeter regime can be used to estimate the redshift of the source. The BLAST bands were chosen to optimize constraints on the FIR peak of the bright submillimeter galaxy population at $1<z<4$. A Monte Carlo photometric redshift method has been developed (Hughes et al. 


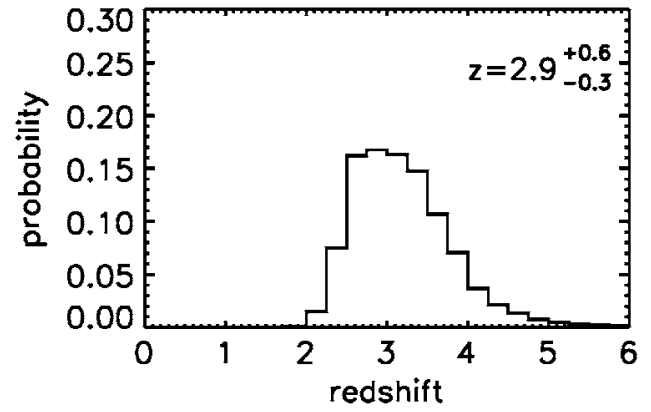

Figure 3. Redshift probability distribution based on $250-1100 \mu$ m photometry at the millimeter centroid position. The uncertainty in lensing magnification results in a longer high-redshift tail.

2002; Aretxaga et al. 2003) to take into account constraining prior information such as a range of local SED templates and the evolving luminosity function to $z \approx 2$.

In accordance with this method, we generate SEDs for a catalog of galaxies based on an evolutionary model for the $60 \mu \mathrm{m}$ luminosity function that fits the observed $850 \mu \mathrm{m}$ number counts (e.g., luminosity evolution $\propto(1+z)^{3}$ for $z \lesssim 2$, and no evolution at $z>2$ ) and covers a simulated area of $10 \mathrm{deg}^{2}$. Template SEDs are drawn at random from a library of local starbursts, ULIRGs, and active galactic nuclei (AGNs) to provide FIR-radio fluxes. The SEDs cover a wide range of FIR luminosities $\left(10^{9.0}-10^{12.3} L_{\odot}\right)$ and temperatures $(25-65 \mathrm{~K})$. The photometric and calibration uncertainties of the BLAST and AzTEC measurements are included in the fluxes of the mock galaxies. We adopt a conservative estimate for the amplification of the source $(A=30$; Wilson et al. 2008; Gonzalez et al. 2009), and reject mock galaxies with fluxes below the detection threshold of the survey. The redshift probability distribution of the source is then calculated as the normalized distribution of the redshifts of the mock galaxies in the catalog, weighted by the likelihood of identifying the colors and fluxes of each mock galaxy with those of the source.

Figure 3 shows the results of this analysis indicating a photometric redshift $z_{\text {phot }}=2.9_{-0.3}^{+0.6}$. This estimate is consistent with the IR-color redshift estimation $\left(z_{\text {phot }}^{\mathrm{IR}}=2.7 \pm 0.2\right)$ for the most likely IRAC counterpart (Wilson et al. 2008; Gonzalez et al. 2009). The accuracy of the technique has been experimentally tested for galaxies detected in at least three bands in the rest-frame radio to FIR interval, yielding a typical $1 \sigma$ error $\left\langle\left(z_{\text {phot }}^{\mathrm{MC}}-z_{\text {spec }}\right)^{2}\right\rangle^{1 / 2} \approx 0.25$ over the $0.5 \lesssim z \lesssim 4.0$ regime (Aretxaga et al. 2007).

\subsubsection{Intrinsic Properties}

With an estimate of its redshift, we can derive properties intrinsic to the submillimeter galaxy. Figure 4 shows a singletemperature modified blackbody SED of the form

$$
S_{v}=N\left(v / v_{0}\right)^{\beta} B_{v}(T),
$$

where $S_{v}$ is the flux density, $N$ is the amplitude, $\beta$ is the dust emissivity index, $\nu_{0}$ is fixed at $c / 250 \mu \mathrm{m}$, and $B_{v}(T)$ is the Planck blackbody radiation function for a source with temperature $T$. The function is fitted to the data using a $\chi^{2}$ minimization routine, after subtracting the contribution from SZE and an estimate of the contribution from the brightest midinfrared cluster member galaxy, as discussed previously. The gray curves indicate the $1 \sigma$ range of temperature and amplitudes for the fit estimated from 200 Monte Carlo simulations. The value of $\beta$ is fixed here at $\beta=1.5$, but the fits give very

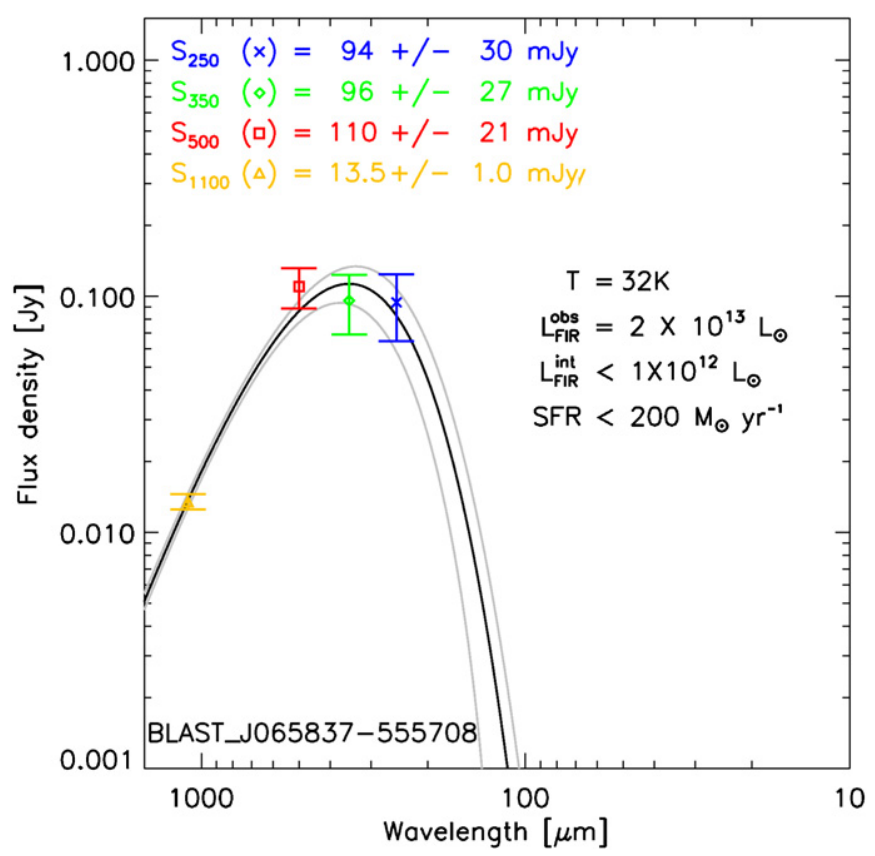

Figure 4. Spectral energy distribution of BLAST J065837-555708. The black curve is the best-fit model to the BLAST and AzTEC measurements, shown on the plot with $1 \sigma$ error bars. These flux densities have been corrected for the contribution from SZE. The gray curves indicate the $1 \sigma$ range of temperature and amplitudes for the fit. The spectral index is fixed at $\beta=1.5$. The spectral information enables a color correction to the measured flux densities which accounts for the profiles of the BLAST filters. These color-corrected values are displayed on the plot. $L_{\mathrm{FIR}}^{\mathrm{obs}}$ represents the far-infrared luminosity based on the fit to observations, while $L_{\mathrm{FIR}}^{\mathrm{int}}$ is an estimate of the intrinsic far-infrared luminosity of the source, assuming an amplification of $A>30$ due to gravitational lensing. An upper limit on the star formation rate is derived based on this luminosity (Kennicutt 1998)

(A color version of this figure is available in the online journal.)

similar values of $T$ and $L_{\mathrm{FIR}}$ if we use $\beta=2.0$. The bestfit temperature of the distant lensed source is $32 \mathrm{~K}$. This SED is consistent with those fit to SMGs observed in regular blank-field surveys (Chapman et al. 2005; Kovács et al. 2006; Aretxaga et al. 2007; Dye et al. 2009). This spectral information also enables a color correction to the flux densities which accounts for the profiles and widths of the BLAST filters (Truch et al. 2008). These color-corrected flux densities are given in Table 4. $L_{\mathrm{FIR}}^{\mathrm{obs}}$ is the FIR luminosity, calculated in the wavelength range $\lambda=8-1000 \mu \mathrm{m}$ (Kennicutt 1998), for the observed flux densities without considering gravitational lensing effects. Using the previously assumed lower limit for the amplitude of the lensing magnification $(A>30)$, we derive an upper limit for the intrinsic FIR luminosity of $L_{\mathrm{FIR}}^{\text {int }}<10^{12} L_{\odot}$. This value is consistent with estimates presented by the AzTEC team (Wilson et al. 2008) as well as from extrapolation of the $24 \mu \mathrm{m}$ luminosity (Gonzalez et al. 2009), and qualifies the source as a LIRG. This intrinsic luminosity implies an upper limit on the star formation rate of $200 M_{\odot} \mathrm{yr}^{-1}$ (Kennicutt 1998). The source is relatively faint compared to typical submillimeterselected galaxies with similar dust temperatures (Blain et al. 2003; Kovács et al. 2006; Dye et al. 2009), and is more consistent with the luminosity-temperature relationship observed in IRASselected samples (Soifer et al. 1991; Blain et al. 2003; Chapman et al. 2003; Chapin et al. 2009).

\section{CONCLUSIONS}

BLAST submillimeter maps of the Bullet Cluster have revealed bright emission near the peak of the weak lensing 
contours (Clowe et al. 2006). The position of the detection coincides with the AzTEC 1.1 mm source MMJ065837-5557.0 and a multiply imaged LIRG detected in the Spitzer IRAC maps (Wilson et al. 2008; Gonzalez et al. 2009). The systematic offsets in peak emission between the BLAST bands motivates the likely interpretation that multiple sources are confused at BLAST resolution. We have performed a simultaneous fit to the brightest two infrared galaxies in the field in order to estimate the properties of the background LIRG. The resulting colorcorrected flux densities for the source are $S_{250}=94 \pm 30$, $S_{350}=96 \pm 27$, and $S_{500}=110 \pm 21 \mathrm{mJy}$.

The BLAST colors sample the peak of the source spectrum, and 250-1100 $\mu \mathrm{m}$ flux densities place strong constraints on its photometric redshift. Using a Monte Carlo photometric redshift method (Hughes et al. 2002; Aretxaga et al. 2003), we have calculated a redshift $z_{\text {phot }}=2.9_{-0.3}^{+0.6}$. The redshift probability distribution is inconsistent with the redshift of the Bullet Cluster $(z \approx 0.3)$, ruling out the possibility that the millimeter AzTEC source is associated with the foreground mass. Assuming the best-fit photometric redshift we have derived the observed FIR luminosity of the source, in the absence of lensing, to be $L_{\mathrm{FIR}}^{\mathrm{obs}}=2 \times 10^{13} L_{\odot}$. The proximity of the source to a critical line in the lensing model (Bradač et al. 2006; Gonzalez et al. 2009) puts a lower limit $A>30$ on the amplitude of magnification due to gravitational lensing by the cluster. This imposes an upper limit on the intrinsic luminosity of the SMG, $L_{\mathrm{FIR}}^{\text {int }}<10^{12} L_{\odot}$. This luminosity is consistent with estimates from Wilson et al. (2008) and Gonzalez et al. (2009), and characterizes the source as a LIRG, a member of the fainter population of SMGs that dominate the number counts but are difficult to detect with the limited resolution of existing submillimeter telescopes.

The challenges in interpreting the BLAST maps demonstrate the necessity of including complementary, higher resolution data sets in the analysis. BLAST is a precursor to the Spectral and Photometric Imaging Receiver (SPIRE) on the recently launched Herschel Space Observatory (Griffin et al. 2006). With nearly twice the resolution, and gains in sensitivity, SPIRE will be able to provide significantly better centroiding accuracy. Nevertheless, access to infrared and millimeter data sets will still be integral to a more complete understanding of these crowded systems.

We acknowledge the support of NASA through grant NAG512785, NAG5- 13301, and NNGO-6GI11G, the NSF Office of Polar Programs, the Canadian Space Agency, the Natural Sciences and Engineering Research Council (NSERC) of Canada, and the UK Science and Technology Facilities Council (STFC). We thank Tony Mroczkowski for his help with the SZE simulations. This research has been enabled by the use of WestGrid computing resources. This research also made use of the SIMBAD database, observations made with the Spitzer Space
Telescope, which is operated by the Jet Propulsion Laboratory, California Institute of Technology under a contract with NASA, and SAOImage DS9, developed by Smithsonian Astrophysical Observatory.

\section{REFERENCES}

Aretxaga, I., Hughes, D. H., Chapin, E. L., Gaztañaga, E., Dunlop, J. S., \& Ivison, R. J. 2003, MNRAS, 342, 759

Aretxaga, I., et al. 2007, MNRAS, 379, 1571

Austermann, J. E., et al. 2009, AzTEC Half Square Degree Survey of the SHADES Fields. I. Maps, Catalogues, and Source Counts, arXiv:0907.1093

Barger, A. J., Cowie, L. L., Sanders, D. B., Fulton, E., Taniguchi, Y., Sato, Y., Kawara, K., \& Okuda, H. 1998, Nature, 394, 248

Bertoldi, F., et al. 2007, ApJS, 172, 132

Blain, A. W., Barnard, V. E., \& Chapman, S. C. 2003, MNRAS, 338, 733

Bradač, M., et al. 2006, ApJ, 652, 937

Chapman, S. C., Blain, A. W., Smail, I., \& Ivison, R. J. 2005, ApJ, 622, 772

Chapman, S. C., Helou, G., Lewis, G. F., \& Dale, D. A. 2003, ApJ, 588, 186

Chapin, E. L., Hughes, D. H., \& Aretxaga, I. 2009, MNRAS, 393, 653

Clowe, D., Bradač, M., Gonzalez, A. H., Markevitch, M., Randall, S. W., Jones, C., \& Zaritsky, D. 2006, ApJ, 648, L109

Clowe, D., Gonzalez, A., \& Markevitch, M. 2004, ApJ, 604, 596

Coppin, K., et al. 2006, MNRAS, 372, 1621

Devlin, M. J., et al. 2009, Nature, 458, 737

Dye, S., et al. 2009, ApJ, 703, 285

Gonzalez, A. H., Clowe, D., Bradač, M., Zaritsky, D., Jones, C., \& Markevitch, M. 2009, ApJ, 691, 525

Greve, T. R., Ivison, R. J., Bertoldi, F., Stevens, J. A., Dunlop, J. S., Lutz, D., \& Carilli, C. L. 2004, MNRAS, 354, 779

Griffin, M., et al. 2006, Proc. SPIE, 6265, 62650A

Hughes, D. H., et al. 1998, Nature, 394, 241

Hughes, D. H., et al. 2002, MNRAS, 335, 871

Itoh, N., Kohyama, Y., \& Nozawa, S. 1998, ApJ, 502, 7

Kennicutt, R. C. Jr. 1998, ARA\&A, 36, 189

Knudsen, K. K., van der Werf, P. P., \& Kneib, J.-P. 2008, MNRAS, 384 1611

Kovács, A., Chapman, S. C., Dowell, C. D., Blain, A. W., Ivison, R. J., Smail, I., \& Phillips, T. G. 2006, ApJ, 650, 592

Makovoz, D., Moshir, M., Laher, R., \& Marsh, K. 2002, in ASP Conf. Ser. 281, Astronomical Data Analysis Software and Systems XI, APEX: A Point Source Extractor for SIRTF, ed. D. A. Bohlender, D. Durand, \& T. H. Handley (San Francisco, CA: ASP), 417

Markevitch, M., Gonzalez, A. H., David, L., Vikhlinin, A., Murray, S., Forman, W., Jones, C., \& Tucker, W. 2002, ApJ, 567, L27

Mehlert, D., et al. 2001, A\&A, 379, 96

Nusser, A. 2008, MNRAS, 384, 343

Pascale, E., et al. 2008, ApJ, 681, 400

Pérez-González, P. G., et al. 2005, ApJ, 630, 82

Pope, A., et al. 2006, MNRAS, 370, 1185

Scott, S. E., et al. 2002, MNRAS, 331, 817

Siringo, G., et al. 2009, A\&A, 497, 945

Smail, I., Ivison, R. J., \& Blain, A. W. 1997, ApJ, 490, L5

Soifer, B. T., et al. 1991, ApJ, 381, L55

Truch, M. D. P., et al. 2008, ApJ, 681, 415

Truch, M. D. P., et al. 2009, ApJ, submitted

Tucker, W., et al. 1998, ApJ, 496, L5

Webb, T. M., et al. 2003, ApJ, 587, 41

Wilson, G. W., et al. 2008, MNRAS, 390, 1061

Zemcov, M., Borys, C., Halpern, M., Mauskopf, P., \& Scott, D. 2007, MNRAS, 376,1073 